VoL. 57 (1998) [427-432]

\title{
PF-RINGS OF GENERALISED POWER SERIES
}

\section{ZHONGKUI LIU}

Let $R$ be a commutative ring and $(S, \leqslant)$ a strictly ordered monoid which satisfies the condition that $0 \leqslant s$ for every $s \in S$. We show that the generalised power series ring $\left[\left[R^{S, \leqslant}\right]\right]$ is a PF-ring if and only if $R$ is a PF-ring.

\section{Introduction and Preliminaries}

Let $R$ be a commutative ring. Recall that $R$ is a PF-ring if every projective $R$ module is free. A famous result of Quillen and Suslin independently states that for a field $F$, every finitely generated projective $F\left[x_{1}, \ldots, x_{n}\right]$-module is free. In [1], it was proved that $R\left[\left[x_{1}, \ldots, x_{n}\right]\right]$ is a PF-ring if and only if $R$ is a PF-ring. In this paper, we shall prove that the generalised power series ring $\left[\left[R^{S, \leqslant}\right]\right]$ is a PF-ring if and only if $R$ is a PF-ring, where $(S, \leqslant)$ is a strictly ordered monoid which satisfies the condition that $0 \leqslant s$ for every $s \in S$. As an application, we obtain some new examples of PF-rings.

All rings considered here are commutative with identity. Any concept and notation not defined here can be found in $[5,6,7]$. For a ring $R$, we denote by $U(R)$ and $J(R)$ the multiplicative group of units, and the Jacobson radical of $R$, respectively.

Let $(S, \leqslant)$ be an ordered set. Recall that $(S, \leqslant)$ is Artinian if every strictly decreasing sequence of elements of $S$ is finite, and that $(S, \leqslant)$ is narrow if every subset of pairwise order-incomparable elements of $S$ is finite. Let $S$ be a commutative monoid. Unless stated otherwise, the operation of $S$ shall be denoted additively, and the neutral element by 0 . The following definition is due to $[5,6,7]$.

Let $(S, \leqslant)$ be a strictly ordered monoid (that is, $(S, \leqslant)$ is an ordered monoid satisfying the condition that, if $s, s^{\prime}, t \in S$ and $s<s^{\prime}$, then $s+t<s^{\prime}+t$ ), and $R$ a commutative ring. Let $A=\left[\left[R^{S, \leqslant}\right]\right]$ be the set of all maps $f: S \longrightarrow R$ such that $\operatorname{supp}(f)=\{s \in S \mid f(s) \neq 0\}$ is Artinian and narrow. With pointwise addition, $A$ is an Abelian additive group. For every $s \in S$ and $f_{1}, \ldots, f_{m} \in A$, let $X_{s}\left(f_{1}, \ldots, f_{m}\right)=$ $\left\{\left(u_{1}, \ldots, u_{m}\right) \in S^{m} \mid s=u_{1}+\cdots+u_{m}, f_{1}\left(u_{1}\right) \neq 0, \ldots, f_{m}\left(u_{m}\right) \neq 0\right\}$. It follows from $[6,1.16]$ that $X_{s}\left(f_{1}, \ldots, f_{m}\right)$ is finite. This fact allows us to define the operation of convolution:

$$
(f g)(s)=\sum_{(u, v) \in X_{s}(f, g)} f(u) g(v) .
$$

Received 18th September, 1997

Research supported by National Natural Science Foundation of China 19501007 and 19671063.

Copyright Clearance Centre, Inc. Serial-fee code: 0004-9729/98 \$A2.00+0.00. 
With this operation, and pointwise addition, $A$ becomes a commutative ring, which is called the ring of generalised power series. The elements of $A$ are called generalised power series with coefficients in $R$ and exponents in $S$.

For example, if $S=\mathbb{N}$ and $\leqslant$ is the usual order, then $\left[\left[R^{\mathbb{N}, \leqslant}\right]\right] \cong R[[x]]$, the usual ring of power series. If $S$ is a commutative monoid and $\leqslant$ is the trivial order, then $\left[\left[R^{S, \leqslant}\right]\right]=R[S]$, the monoid-ring of $S$ over $R$. Further examples are given in [5]. Many results on $\left[\left[R^{S, \leqslant}\right]\right]$ have been obtained in $[2,3,4,5,6,7]$.

We shall use the following notations introduced by Ribenboim in [5].

Let $a \in R$. Define a mapping $c_{a} \in\left[\left[R^{S, \leqslant}\right]\right]$ as follows:

$$
c_{a}(0)=a, \quad c_{a}(s)=0, \quad 0 \neq s \in S
$$

Let $s \in S$. Define a mapping $e_{s} \in\left[\left[R^{S, \leqslant]}\right]\right.$ as follows:

$$
e_{s}(s)=1, \quad e_{s}(t)=0, \quad s \neq t \in S
$$

Then $R$ is canonically embedded as a subring of $\left[\left[R^{S, \leqslant}\right]\right]$, and $S$ is canonically embedded as a submonoid of $\left(\left[\left[R^{S, \leqslant}\right]\right]-\{0\}, \bullet\right)$. It is easy to see that $e_{0}$ is the identity of $\left[\left[R^{S, \leqslant}\right]\right]$.

\section{Main Results}

We shall henceforth assume that $(S, \leqslant)$ is a strictly ordered monoid which satisfies the condition:

$$
0 \leqslant s \text { for every } s \in S
$$

LEMMA 2.1. [6] Let $f \in\left[\left[R^{S, \leqslant}\right]\right]$. Then $f \in U\left(\left[\left[R^{S, \leqslant}\right]\right]\right)$ if and only if $f(0) \in$ $U(R)$.

Corollary 2.2. Let $f \in\left[\left[R^{S, \leqslant}\right]\right]$. Then $f$ is in $J\left(\left[\left[R^{S, \leqslant}\right]\right]\right)$ if and only if $f(0)$ is in $J(R)$.

ProOF: Suppose that $f(0) \in J(R)$. Then $1-r f(0) \in U(R)$ for every $r \in R$. For each $g \in\left[\left[R^{S, \leqslant]}\right]\right.$, we have $(g f)(0)=\sum_{(u, v) \in X_{0}(g, f)} g(u) f(v)=g(0) f(0)$ by the condition (S0). Thus $\left(e_{0}-g f\right)(0)=e_{0}(0)-(g f)(0)=1-g(0) f(0) \in U(R)$. By Lemma 2.1, it follows that $e_{0}-g f \in U\left(\left[\left[R^{S, \leqslant}\right]\right]\right)$, which means that $f \in J\left(\left[\left[R^{S, \leqslant}\right]\right]\right)$.

Conversely suppose that $f \in J\left(\left[\left[R^{S, \leqslant}\right]\right]\right)$. For every $r \in R, e_{0}-c_{r} f \in U\left(\left[\left[R^{S, \leqslant}\right]\right]\right)$. Thus, by Lemma $2.1,1-r f(0)=\left(e_{0}-c_{r} f\right)(0) \in U(R)$, and so $f(0) \in J(R)$.

PROPOSITION 2.3. There exists a group isomorphism $K_{0}\left[\left[R^{S, \leqslant}\right]\right] \cong K_{0} R$. 
Proof: Since $(S, \leqslant)$ satisfies the condition (S0), it is easy to see that for any $f, g \in\left[\left[R^{S, \leqslant}\right]\right],(f g)(0)=\sum_{(u, v) \in X_{0}(f, g)} f(u) g(v)=f(0) g(0)$. Thus there exist ring homomorphisms

$$
\begin{aligned}
\alpha:\left[\left[R^{S, \leqslant}\right]\right] & \rightarrow R \\
f & \mapsto f(0)
\end{aligned}
$$

and

$$
\begin{gathered}
\beta: R \longrightarrow\left[\left[R^{S, \leqslant}\right]\right] \\
r \mapsto c_{r}
\end{gathered}
$$

Clearly $\alpha \beta=1_{R}$. Thus $K_{0} \alpha$ is a surjective homomorphism. Let $f \in \operatorname{Ker}(\alpha)$. Then $f(0)=0 \in J(R)$. By Corollary 2.2 , it follows that $f \in J\left(\left[\left[R^{S, \leqslant}\right]\right]\right)$. This means that $\operatorname{Ker}(\alpha) \subseteq J\left(\left[\left[R^{S, \leqslant}\right]\right]\right)$. Thus, by [8, Proposition 9$], K_{0} \alpha$ is a monomorphism. Now the result follows.

We note that the group isomorphism above is also a ring isomorphism since the rings we considered are commutative (see [1]).

A ring $R$ is called a Hermite ring provided for every $\left(r_{1}, \ldots, r_{n}\right) \in R^{n}$, if there exists $\left(p_{1}, \ldots, p_{n}\right) \in R^{n}$ such that $r_{1} p_{1}+\cdots+r_{n} p_{n}=1$, then there exists a $n \times n$ matrix $M$ over $R$ with first row $\left(r_{1}, \ldots, r_{n}\right)$ and $\operatorname{det}(M)$ a unit in $R$.

Proposition 2.4. $\left[\left[R^{S, \leqslant}\right]\right]$ is a Hermite ring if and only if $R$ is a Hermite ring.

Proof: Let $\left[\left[R^{S, \leqslant}\right]\right]$ is a Hermite ring. Suppose that $\left(r_{1}, \ldots, r_{n}\right)$ and $\left(p_{1}, \ldots, p_{n}\right)$ are in $R^{n}$ such that $r_{1} p_{1}+\cdots+r_{n} p_{n}=1$. Since $\left(c_{r_{1}} c_{p_{1}}+\cdots+c_{r_{n}} c_{p_{n}}\right)(s)=$ $\sum_{i=1}^{n}\left(c_{r_{i}} c_{p_{i}}\right)(s)=\sum_{i=1}^{n} \sum_{(u, v) \in X_{s}\left(c_{r_{i}}, c_{p_{i}}\right)} c_{r_{i}}(u) c_{p_{i}}(v)=0=e_{0}(s)$ when $s \neq 0$, and $\left(c_{r_{1}} c_{p_{1}}+\cdots+c_{r_{n}} c_{p_{n}}\right)(0)=\left(c_{r_{1}} c_{p_{1}}\right)(0)+\cdots+\left(c_{r_{n}} c_{p_{n}}\right)(0)=r_{1} p_{1}+\cdots+r_{n} p_{n}=1=$ $e_{0}(0)$, we have

$$
c_{r_{1}} c_{p_{1}}+\cdots+c_{r_{n}} c_{p_{n}}=e_{0}
$$

Since $\left[\left[R^{S, \leqslant}\right]\right]$ is a Hermite ring, there exists a $n \times n$ matrix $M$ over $\left[\left[R^{S, \leqslant}\right]\right]$ with first row $\left(c_{r_{1}}, \ldots, c_{r_{n}}\right)$ and $\operatorname{det}(M)$ a unit in $\left[\left[R^{S, \leqslant}\right]\right]$. Suppose that

$$
M=\left(\begin{array}{cccc}
c_{r_{1}} & c_{r_{2}} & \ldots & c_{r_{n}} \\
f_{21} & f_{22} & \ldots & f_{2 n} \\
\vdots & \vdots & \ddots & \vdots \\
f_{n 1} & f_{n 2} & \ldots & f_{n n}
\end{array}\right)
$$


Denote

$$
N=\left(\begin{array}{cccc}
r_{1} & r_{2} & \ldots & r_{n} \\
f_{21}(0) & f_{22}(0) & \ldots & f_{2 n}(0) \\
\vdots & \vdots & \ddots & \vdots \\
f_{n 1}(0) & f_{n 2}(0) & \ldots & f_{n n}(0)
\end{array}\right)
$$

Since $S$ satisfies the condition (S0), it is easy to see that

$$
\begin{aligned}
\operatorname{det}(M)(0) & =\left(\sum_{i_{1} \ldots i_{n}}(-1)^{\pi\left(i_{1} \ldots i_{n}\right)} c_{r_{i_{1}}} f_{2 i_{2}} \ldots f_{n i_{n}}\right)(0) \\
& =\sum_{i_{1} \ldots i_{n}}(-1)^{\pi\left(i_{1} \ldots i_{n}\right)} r_{i_{1}} f_{2 i_{2}}(0) \ldots f_{n i_{n}}(0)=\operatorname{det}(N)
\end{aligned}
$$

By Lemma 2.1, it follows that $\operatorname{det}(N) \in U(R)$. Thus $R$ is a Hermite ring.

Conversely suppose that $R$ is a Hermite ring. Assume that $\left(f_{1}, \ldots, f_{n}\right)$ and

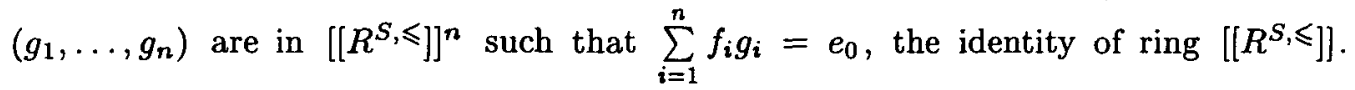
Then

$$
\sum_{i=1}^{n} f_{i}(0) g_{i}(0)=1
$$

Since $R$ is a Hermite ring, there exists a $n \times n$ matrix

$$
P=\left(\begin{array}{cccc}
f_{1}(0) & f_{2}(0) & \ldots & f_{n}(0) \\
r_{21} & r_{22} & \ldots & r_{2 n} \\
\vdots & \vdots & \ddots & \vdots \\
r_{n 1} & r_{n 2} & \ldots & r_{n n}
\end{array}\right)
$$

over $R$ with first row $\left(f_{1}(0), \ldots, f_{n}(0)\right)$ and $\operatorname{det}(P) \in U(R)$. Let

$$
Q=\left(\begin{array}{cccc}
f_{1} & f_{2} & \ldots & f_{n} \\
c_{r_{21}} & c_{r_{22}} & \ldots & c_{r_{2 n}} \\
\vdots & \vdots & \ddots & \vdots \\
c_{r_{n 1}} & c_{r_{n 2}} & \cdots & c_{r_{n n}}
\end{array}\right)
$$

Then, by condition (S0), it is easy to see that $(\operatorname{det}(Q))(0)=\operatorname{det}(P) \in U(R)$. Thus, by Lemma 2.1 , it follows that $\operatorname{det}(Q) \in U\left(\left[\left[R^{S, \leqslant}\right]\right]\right)$. This means that $\left[\left[R^{S, \leqslant}\right]\right]$ is a Hermite ring.

Now we have: 
THEOREM 2.5. Let $(S, \leqslant)$ be a strictly ordered monoid which satisfies the condition that $0 \leqslant s$ for every $s \in S$. Then $\left[\left[R^{S, \leqslant}\right]\right.$ is a $P F$-ring if and only if $R$ is a $P F$-ring.

Proof: It is well-known that a commutative ring $A$ is a PF-ring if and only if $A$ is a Hermite ring and there exists a ring isomorphism $K_{0} A \cong \mathbb{Z}$ (see, for example, [9]). Thus the result follows from Proposition 2.3 and 2.4.

CoRollary 2.6. [1] $R\left[\left[x_{1}, \ldots, x_{n}\right]\right]$ is a PF-ring if and only if $R$ is a PF-ring.

Proof: Let $S=\mathbb{N} \times \cdots \times \mathbb{N}$ ( $n$ copies) with the product of the usual order. Then $\left[\left[R^{S, \leqslant}\right] \cong R\left[\left[x_{1}, \ldots, x_{n}\right]\right]\right.$. Now the result follows from Theorem 2.5 .

The following corollaries will give other examples of PF-rings.

Corollary 2.7. Let $\mathbb{Q}^{+}=\{a \in \mathbb{Q} \mid a \geqslant 0\}, \mathbb{R}^{+}=\{a \in \mathbb{R} \mid a \geqslant 0\}$. Then the rings $\left[\left[\mathbb{Z}^{\mathbb{N}, \leqslant}\right]\right],\left[\left[\mathbb{Z}^{\mathbb{Q}^{+}}, \leqslant\right]\right]$and $\left[\left[\mathbb{Z}^{\mathbb{R}^{+}} \leqslant\right]\right]$are PF-rings, where $\leqslant$is the usual order.

COROLLARY 2.8. Let $\left(S_{1}, \leqslant_{1}\right), \ldots,\left(S_{n}, \leqslant_{n}\right)$ be strictly ordered monoids which satisfy the condition that $0 \leqslant i s$ for every $s \in S_{i}, i=1, \ldots, n$. Denote by (lex $\leqslant$ ) and (revlex $\leqslant$ ) the lexicographic order, the reverse lexicographic order, respectively, on the monoid $S_{1} \times \cdots \times S_{n}$. Then $R$ is a PF-ring if and only if $\left[\left[R^{S_{1} \times \cdots \times S_{n},(l e x \leqslant)}\right]\right]$ is a $P F$-ring if and only if $\left[\left[R^{S_{1} \times \cdots \times S_{n},(\text { revlex } \leqslant)}\right]\right]$ is a $P F$-ring.

Proof: It is easy to see that $\left(S_{1} \times \cdots \times S_{n},(l e x \leqslant)\right)$ is a strictly ordered monoid which satisfies the condition that $(0, \ldots, 0)(l e x \leqslant)\left(s_{1}, \ldots, s_{n}\right)$ for every $\left(s_{1}, \ldots, s_{n}\right) \in$ $S_{1} \times \cdots \times S_{n}$. Thus, by Theorem $2.5, R$ is a PF-ring if and only if $\left[\left[R^{S_{1} \times \cdots \times S_{n},(l e x \leqslant)}\right]\right]$ is a PF-ring.

The proof of the another assertion is similar.

Let $R$ be a ring, and consider the multiplicative monoid $\mathbb{N}_{\geqslant 1}$, endowed with the usual order $\leqslant$. Then $A=\left[\left[R^{\mathbb{N}} \geqslant 1, \leqslant\right]\right]$ is the ring of arithmetical functions with values in $R$, endowed with the Dirichlet convolution:

$$
(f g)(n)=\sum_{d \mid n} f(d) g(n / d), \quad \text { for each } n \geqslant 1
$$

Corollary 2.9. $\left[\left[R^{\mathbb{N} \geqslant 1, \leqslant}\right]\right]$ is a $P F$-ring if and only if $R$ is a PF-ring.

\section{REFERENCES}

[1] Chen Huanyin, 'Some results on Grothendieck groups', J. Nanjing Univ. 31 (1995), 1-8.

[2] G.A. Elliott and P. Ribenboim, 'Fields of generalized power series', Arch. Math. 54 (1990), 365-371.

[3] P. Ribenboim, 'Generalized power series', in Lattices, semigroups and universal algebra, (J. Almeida, G. Bordalo, and P. Dwinger, Editors) (Plenum, New York, 1990). 
[4] P. Ribenboim, 'Rings of generalized power series: Nilpotent elements', Abh. Math. Sem. Univ. Hamburg 61 (1991), 15-33.

[5] P. Ribenboim, 'Noetherian rings of generalized power series', J. Pure Appl. Algebra 79 (1992), 293-312.

[6] P. Ribenboim, 'Rings of generalized power series II: units and zero-divisors', J. Algebra 168 (1994), 71-89.

[7] P. Ribenboim, 'Special properties of generalized power series', J. Algebra 173 (1995), 566-586.

[8] J.R. Silvester, Introduction to Algebraic K-Theory (Chapman and Hall, London, New York, 1981).

[9] Tong Wenting, 'Grothendieck groups and their applications', J. Math. Nanjing Univ. 3 (1986), 1-11.

Department of Mathematics

Northwest Normal University

Lanzhou 730070

People's Republic of China 